\title{
Tissue Morphology and Cell Impedance Based Biosensors for Toxicity Testing
}

\author{
S. Ďad'o \\ Faculty of Electrical Engineering, CTU Prague, Czech Republic, email: dado@fel.cvut.cz
}

\begin{abstract}
In vitro neurotoxicity testing and toxicity effect quantification plays an important role in many disciplines of biomedicine as an alternative to in vivo methods. The principle of the majority of in vitro methods corresponds to the basic concept of biosensors, i.e. measured quantity is by means of biological sensing element transformed to physical quantity easily measurable by electrical methods of measurement. Two types of biosensors suitable for neurotoxicity measurements are described in the paper. A common feature for both types is application of a living organism as biological sensing element. In the first type of biosensor the morphology of cell is evaluated using image processing methods known as videometry. In the second type of biosensors the electrical impedance of cells using an improved version of the ECIS (Electric Cell-substrate Impedance Sensing) method is a measure of toxicity effects. The results of experiments with biosensors using videometry and a proposal for improvements of ECIS based biosensors are included in the paper.
\end{abstract}

Keywords: In vitro neurotoxicity testing, Biosensors, Impedance of cells, ECIS method

\section{INTRODUCTION}

$\mathrm{N}$ EUROTOXICITY is defined as any adverse effect on the chemistry, structure and function of the nervous system during development or at the maturity induced by chemical or physical influences. For both economic and humane considerations there has been growing interest in alternatives to the use of animals in toxicity testing of chemical agents. Tissue culture has the potential to replace animal testing, but for the success of in vitro approaches new and sensitive methods to detect tissue activities are required.

For the detection of tissue (cellular) activities the concept of biosensors can be used. As it is well known, biosensors (Fig.1)

are devices incorporating a biological sensing element coupled to a variety of transducers (sensors) which convert a biological interaction into a relatively easily measurable electrical signal. Neurotoxicity testing by videometric methods uses spinal ganglions as biological sensing elements. Spinal ganglions are clusters of nerve cells - neurons with nerve fibre sprouts called neuritis (Fig.2.). The change of neuritis morphology is a very sensitive indicator of toxicity of the tested substance.

Typical biological sensing elements for testing toxicity by impedance methods (ECIS) are fibroblastic V79 cells. The measure of toxicity is the time response function of impedance after cells are exposed to toxicants.

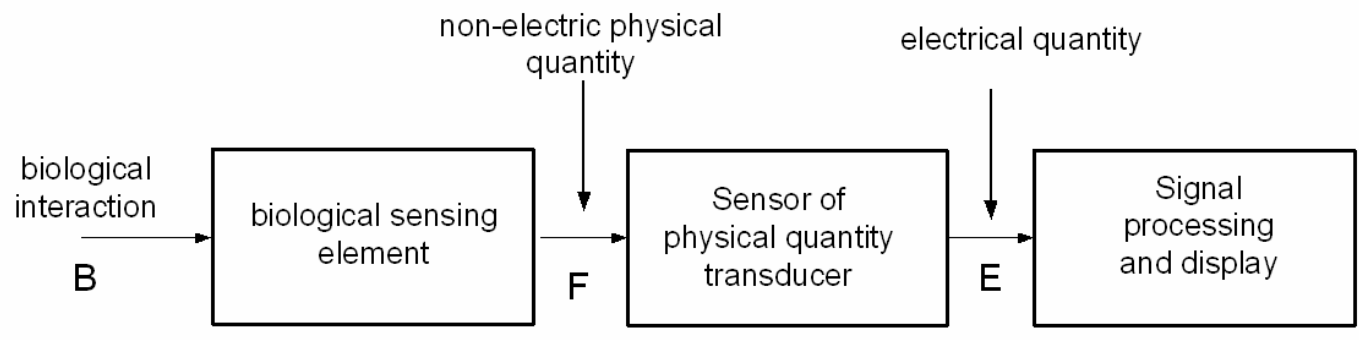

Fig.1 Block diagram of the typical biosensor
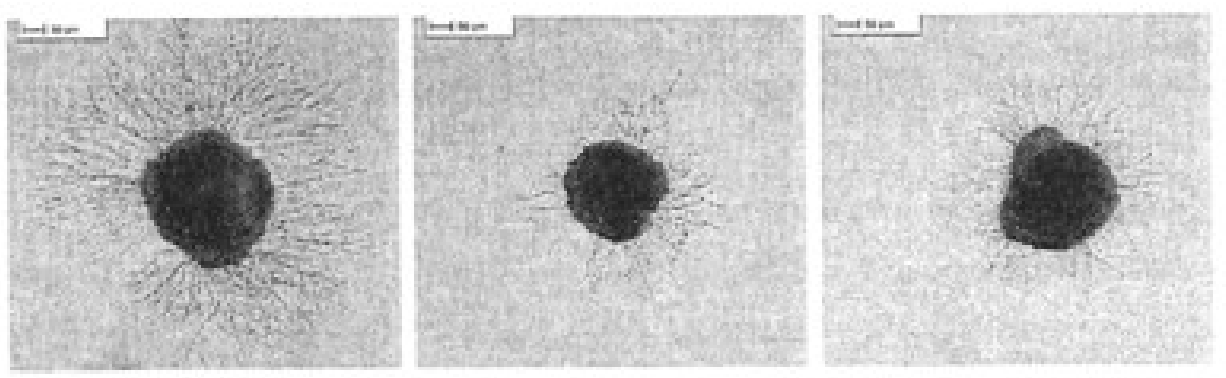

Fig.2 The morphological changes of ganglion caused by toxic substances.

(On the left - reference ganglion, in the middle - ganglion damaged by the effect of TPPS4, on the right - the effect of Photosan). 


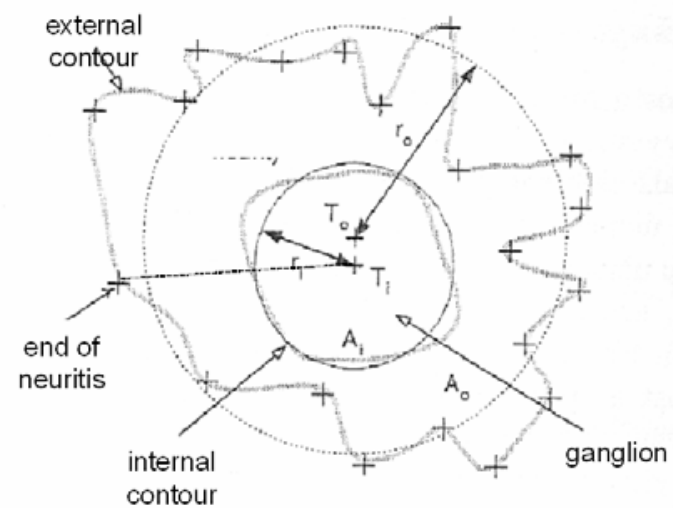

Fig.3 Internal and external contours
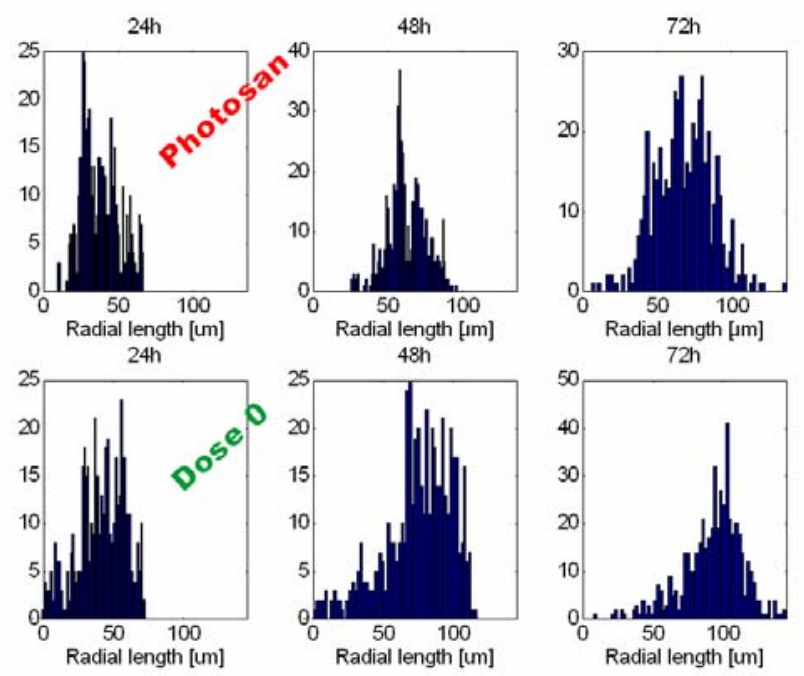

Fig.4 Histograms of the radial length of neuritis.

Dose 0- reference, upper row ganglions affected by Photosan

\section{BIOSENSORS BASED ON IMAGE PROCESSING (VIDEOMETRY)}

The degree of toxicity is estimated from morphological changes of neuritis exposed to toxic substance. The goal is to find quantitative parameters of ganglion geometry satisfactorily expressing the degree of toxicity of the tested substance. The changes of the radial length of neuritis (Fig.4) can be used as a measure of toxicity [1]. Another possibility is an analysis of contours. In the case of ganglion the external contour is an outline connecting the endpoints of neuritis and the internal contour is a circle approximating the ganglion shape (Fig.3).

\section{Methods based on analysis of contours}

Describing the contour by equation in polar coordinates analysis the discrete Fourier transform (DFT) or wavelet transform (DWT) can be used. By interpolation between endpoints we obtain continuous function $c(\varphi)$ in polar coordinates. Then continuous wavelet transform of function $f(t)$ defined by Equ. 1 can be used

$$
W f(\tau, s)=\int_{-\infty}^{+\infty} f(t) \frac{1}{\sqrt{s}} \bar{\psi}\left(\frac{t-\tau}{s}\right) d t
$$

where $s$ is scale (amplitude) parameter and $\tau$ determines position of mother wavelet $\psi(t)$ on time scale. WT with properly chosen mother wavelet allows description of contour by minimum number of coefficients [2].

\section{Methods based on descriptor CWT}

In this approach the contour is divided to $\mathrm{N}$ segments having length $k$ and for each segment transformation (1) is applied. The descriptor CWT is then defined by relation

$$
\operatorname{CDc}(\tau, s)=\sum_{k=1}^{N} \int_{k}^{k+1} c(k) \frac{1}{\sqrt{s}} \psi\left(\frac{t-\tau}{s}\right) d t
$$

\section{Descriptions using skeleton}

By plotting local maximums of absolute values of descriptors in coordinate $\tau$ for each particular scale $s$, the so called skeleton (Fig.5, on the right) is created.

The advantage of skeleton description is simple elimination of noise (stochastic variation of radial length). The noise causes the appearance of skeleton line for small values of $s$ which are then easily separated from long lines-attributes of contour [3].
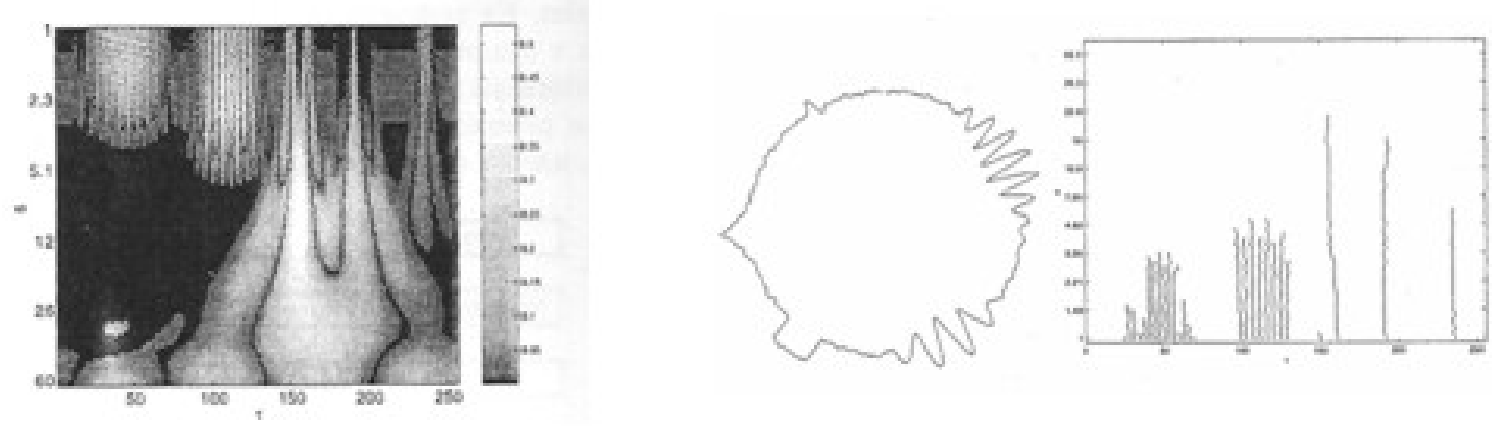

Fig.5 Left: 3-D graph of WT coefficients (wavelet Mexican hat) Middle: simulation of contour radial noise. Right: skeleton lines - ridges of maxima 


\section{BIOSENSORS BASED ON MEASUREMENT OF CELL IMPEDANCE}

In this case the biological sensing element is a monolayer of cells between electrodes located in wells filled with culture medium (Fig.6). The presence of cells increases impedance from the original one when only culture medium is present due to the insulation properties of cell membranes. Ideally, the effect of individual cell on the impedance should be observable (spatial resolution).

The arrangement of electrodes using "spread resistance" principle (known from semiconductor resistivity measurement or from mercury drop polarography) can fulfill this task. Two (gold) electrodes, a miniature active electrode (diameter typically $250 \mu \mathrm{m}$ ) and a large reference one (area approx. 300 times larger) are used. The current density through active electrode is much higher, thus only processes in its vicinity affect the impedance.

The electrode with small diameter is created by covering the gold foil in insulation film and leaving only a "window" of appropriate size (Fig.8) uncovered. Impedance is measured with a weak AC signal $(1 \mu \mathrm{A})$ in frequency range from 10 to $10^{5} \mathrm{~Hz}$. When cells attach and spread on and between electrodes, their insulating membranes constrain the current, forcing it to flow beneath and between the cells. A limited population of cells $(1$ to 1,000) is measured at the time. This results in impedance changes that can be readily measured and used to quantify cell behaviour.

Capacitance part of impedance has maximum value when the well is filled by conductive culture medium only. Then the insulation film represents the dielectric of the "capacitor" in which the conductive culture medium behaves as an electrode connected to the reference electrode. When cells proliferate, the gap between electrodes increases and capacity decreases (Fig.7)

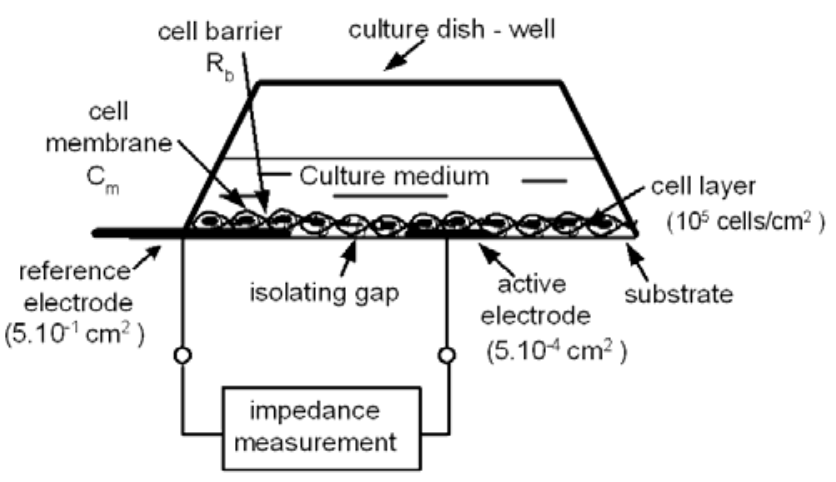

Fig.6 The principle of ECIS [4] (Patent US 7,399,631 B2)

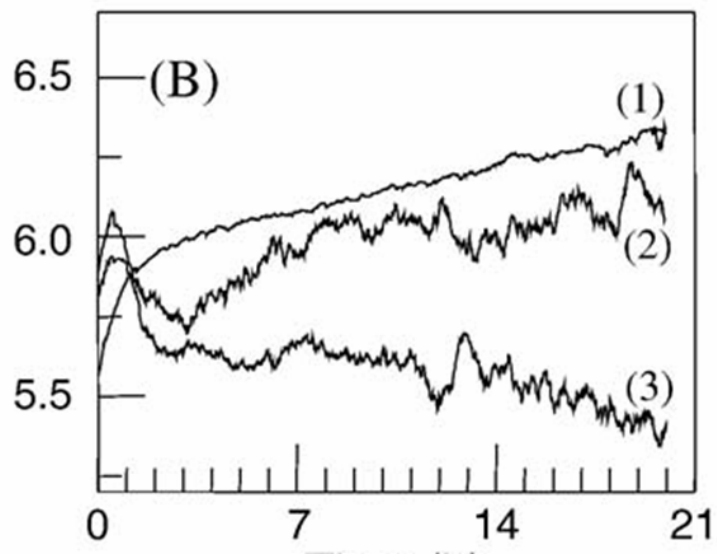

Fig.7 The change of $\mathrm{C}_{\mathrm{m}}[\mathrm{nF}]$ with time $[\mathrm{h}](\mathrm{f}=4 \mathrm{kHz})$ (line 1-no cells, line 2,3-cell culture of V79)

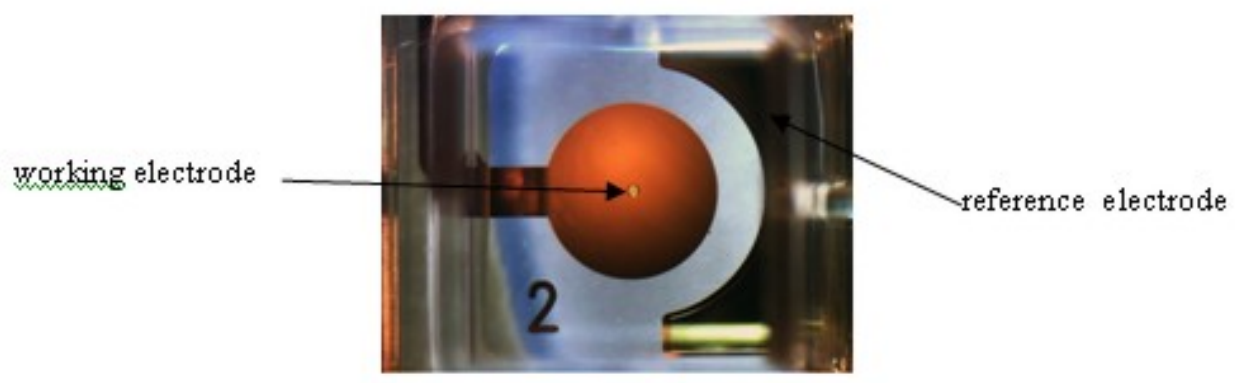

Fig.8 The basic shape of electrodes

\section{RESULTS AND CONCLUSIONS}

Biosensors for toxicity testing using morphological changes of cells are the most widespread methods of in vitro toxicity testing. The transduction of cell morphology to image processing (videometry) offers more information, but requires complicated instrumentation and signal processing. The biosensors transforming cell morphology changes to impedance are much simpler and less demanding. They are just at the beginning of the era of their application in toxicology and represent the perspective orientation of in vitro toxicity testing.
But in both approaches finding the "universal" toxicity quantification parameter and method is extremely difficult, mainly due to problems with time demands and health risks during experimental activities necessary for verification of the proposed toxicity criterion. At present the choice of toxicity testing procedure depends to a large extent on the type of toxic agent. 


\section{ACKNOWLEDGEMENTS}

This work was supported by the research project MSM ČR 6840770012 "Transdisciplinary research in the domain of biomedical engineering".

The author would like to thank Prof. V. Mandys, from the 1st Department of Pathology, Charles University, Prague, for valuable information and cooperation on experiments.

\section{REFERENCES}

[1] Ďad'o, S., Mandys, V., Šmíd, R. (2001). Biomedical shape measurement for neurotoxicity testing. Measurement Science Review, 1 (1), 55-58.
[2] Ďad'o, S., Šmíd, R. (1998). Characterization of an object shape in the picture using continuous wavelet transform. In Proceedings of New Orientations in Signal Processing. Liptovský Mikuláš: Military Academy, 3-6. (in Czech)

[3] Ďad'o, S., Šmíd, R. (2003). Wavelet correlation for biomedical shape evaluation. In Measurement 2003 : Proceedings of the 4th International Conference on Measurement. Bratislava: Slovak Academy of Sciences, Institute of Measurement Science, 256-259.

[4] Keese, C., Karra, N., Dillon, B., Goldberg, A., Giaever, I. (1998). Cell-substratum interactions as a predictor of cytotoxicity. Vitro \& Molecular Toxicology, 11 (2), 183-192. 